\title{
Antibacterial activity of royal jelly-mediated green synthesized silver nanoparticles
}

\author{
Susanna Gevorgyan ${ }^{1}$, Robin Schubert², Mkrtich Yeranosyann 3,4, Lilit Gabrielyan' ${ }^{1}$ Armen Trchounian', \\ Kristina Lorenzen ${ }^{2}$ and Karen Trchounian ${ }^{1 *}$ (D)
}

\begin{abstract}
The application of green synthesis in nanotechnology is growing day by day. It's a safe and eco-friendly alternative to conventional methods. The current research aimed to study raw royal jelly's potential in the green synthesis of silver nanoparticles and their antibacterial activity. Royal jelly served as a reducing and oxidizing agent in the green synthesis technology of colloidal silver nanoparticles. The UV-Vis maximum absorption at $\sim 430 \mathrm{~nm}$ and fluorescence emission peaks at $\sim 487 \mathrm{~nm}$ confirmed the presence of Ag NPs. Morphology and structural properties of Ag NPs and the effect of ultrasound studies revealed: (i) the formation of polydispersed and spherical particles with different sizes; (ii) size reduction and homogeneity increase by ultrasound treatment. Antibacterial activity of different concentrations of green synthesized Ag NPs has been assessed on Gram-negative S. typhimurium and Gram-positive S. aureus, revealing higher sensitivity on Gram-negative bacteria.
\end{abstract}

Keywords: Silver nanoparticles, Green synthesis technology, Royal jelly, Antibacterial activity

\section{Introduction}

Nanoscience is a remarkable field that has the highest potential to improve human life (Ismail et al. 2018; Trchounian et al. 2018). In recent years, nanomaterials' widespread application in various fields of industry, technology, and medicine led to the exponential growth of their global demand (Rónavári et al. 2017; Gabrielyan and Trchounian 2019). Among different metal nanoparticles (NPs), the yearly increase in silver (Ag) NP production was estimated to be hundreds of tons worldwide. The high demand for Ag NPs is conditioned by the commercial utilization of Ag NPs in optics, electronics, catalysis, household items, and a broad range of medical applications (Ge et al. 2014; Gabrielyan and Trchounian 2019). Ag NPs are known for their broad-spectrum antimicrobial and antiviral activities (Gabrielyan and Trchounian 2019; Gabrielyan et al. 2020). These NPs are widely

\footnotetext{
${ }^{*}$ Correspondence: k.trchounian@ysu.am

${ }^{1}$ Department of Biochemistry, Microbiology and Biotechnology, Yerevan State University, Alex Manoogian 1, 0025 Yerevan, Armenia Full list of author information is available at the end of the article
}

applied in different industries in the disinfection of ailments, water, medical instruments, etc. Also, Ag NPs can play an essential role in preventing epidemics caused by progressive drug-resistant pathogens (Lara et al. 2011).

Metal NPs synthesis methods are classified as chemical, physical and biochemical synthesis methods (Fig. 1) (Vishwarsrao et al. 2019). Chemical- and physical-based synthesis techniques usually involve toxic organic solvents and hazardous reagents that carry environmental and biological risks and can influence nanoparticle properties, such as toxicity, thus limiting their application in medicine (Forough et al. 2010; Salem et al. 2020). From this point of view, the development of environmentally friendly processes for the synthesis of metal NPs is of great interest.

Green synthesis (GS) strategies of metal NPs are safer alternatives to the conventional chemical and physical methods with a low environmental footprint and mild experimental conditions. It includes mild temperature and pressure and non-toxic, environmentally benign solvents, reducing agents, and capping materials (Kozma et al. 2016; Thunugunta et al. 2015; Narayanan et al.
Springer Open

(c) The Author(s) 2021. This article is licensed under a Creative Commons Attribution 4.0 International License, which permits use, sharing, adaptation, distribution and reproduction in any medium or format, as long as you give appropriate credit to the original author(s) and the source, provide a link to the Creative Commons licence, and indicate if changes were made. The images or other third party material in this article are included in the article's Creative Commons licence, unless indicated otherwise in a credit line to the material. If material is not included in the article's Creative Commons licence and your intended use is not permitted by statutory regulation or exceeds the permitted use, you will need to obtain permission directly from the copyright holder. To view a copy of this licence, visit http://creativecommons.org/licenses/by/4.0\%. 


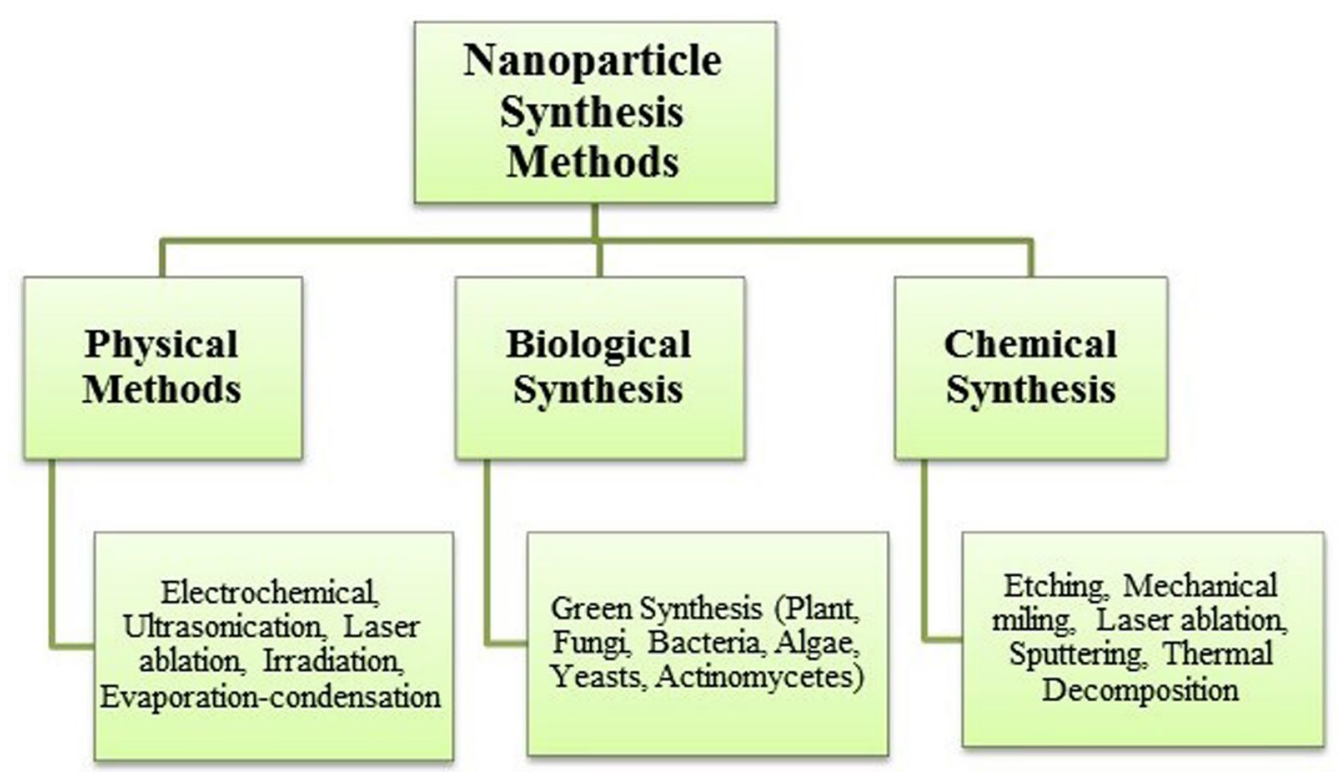

Fig. 1 Nanoparticle synthesis methods

2011). GS can involve living organisms, such as bacteria (Joerger et al. 2000) or fungi (Moghaddam et al. 2015) and plants or natural extracts containing various biologically active compounds like polysaccharides, proteins, vitamins, or alkaloids. The advantage of listed compounds is that they are generally non-toxic, biodegradable, and can act both as reducing and capping agents, thereby promoting the formation and inhibiting the agglomeration of NPs (Moghaddam et al. 2015; Rónavári et al. 2017).

Venu et al. (2011) described the use of aqueous honey solutions in the synthesis of nanomaterials. From this point of view, the royal jelly (RJ) is an attractive honeybee product. It consists of water $(50-60 \%)$, proteins (18\%), carbohydrates (15\%), lipids (3-6\%), mineral salts (1.5\%), and vitamins (Nagai and Inoue 2004). Also, RJ is composed of many bioactive compounds (Sugiyama et al. 2012). Many studies reported various activities of RJ such as pharmacological activities including antitumor (Swellam et al. 2003), antioxidative (Guo et al. 2007), antimicrobial (Romanelli et al. 2011), vasodilative and hypotensive, anti-fatigue, and anti-allergy, antihypercholesterolemic and anti-inflammatory activities (Fratini et al. 2016). In addition to several physiological effects, RJ is widely used in commercial medical products and food industry (Guo et al. 2007).

RJ's composition is relatively constant at the macrolevel. Still, it can vary depending on various factors such as bee nutrition, bee species, harvesting method, the age of the bee larvae, and floral variety, geographical and environmental conditions (Kolayli et al. 2015). From this viewpoint, Armenian honeybee products, including RJ, have unique advantages conditioned by Armenia's geographical, climatic conditions, and plant diversity.

This study is aimed to evaluate Armenian RJ's potential as a source of oxidizing and reducing agents in the GS of Ag NPs. In addition to physical-chemical characteristics determined by using UV-Vis and fluorescent spectroscopy, scanning electron microscopy (SEM), transmission electron microscopy (TEM), selected area electron diffraction (SAED), the antibacterial activity of RJ-mediated GS Ag NPs has been assessed on Salmonella typhimurium MDC1759 and Staphylococcus aureus MDC5233 strains.

\section{Materials and methods Ag NPs synthesis}

Raw royal jelly (RRJ) was obtained from the local Armenian beekeeping factory (Province Kotayk, Armenia). As a source of Ag, silver nitrate solution was used. All solutions during the experiment were prepared in doubly distilled water.

For the synthesis of Ag NPs, RRJ aqueous solution in $0.1 \mathrm{~g} \mathrm{~mL}^{-1}$ concentration and $0.5 \mathrm{M}$ silver nitrate solution were used. 1:1 ratio of each solution was mixed under stirring and kept up to $24 \mathrm{~h}$ on a magnetic stirrer at room temperature (Mendoza-Reséndez et al. 2014). During the synthesis, the solution's color started to change from yellowish to brownish color, which indicates the reduction of $\mathrm{Ag}^{+}$. The final solution was centrifuged and washed with double distilled water several times. Ag NPs were dispersed in double distilled water using ultrasound 
(US) - treatment for $20 \mathrm{~min}$ (Power of US-homogenizer-50 W; YaXun 2000A).

\section{Ag NPs characterization}

The optical absorption spectrum of Ag NPs was obtained using UV-Vis spectrophotometer (GENESYS 10S UVVis, Thermo Scientific, USA) at a resolution of $1 \mathrm{~nm}$ between 280 and $720 \mathrm{~nm}$ ranges. Fluorescent emission spectra were recorded using a Fluorescence spectrophotometer (Agilent Technologies, USA) at CANDLE Synchrotron Research Institute (Yerevan, Armenia) at different excitation wavelengths.

Morphology, microstructure, and size of GS NPs were observed by scanning electron microscopy (SEM, Leibniz Institute of Photonic Technology, Germany) with $15 \mathrm{kV}$ accelerating voltage, $\times 15,000-70,000$ magnification, secondary electron imaging (SEI) and transmission electron microscopy (JEM-2100-Plus, JEOL, Germany). For TEM measurements, the sample was placed on glow discharged carbon-coated copper grids, incubated, blotted, and dried. Transmission electron micrographs were taken using an accelerating voltage of $200 \mathrm{kV}$. Selected area electron diffraction (SAED) of the NPs was also recorded using the TEM. TEM experiments were conducted in the XBI Biolab of European XFEL (Han et al. 2021). Average Ag core diameter, size distributions were calculated for each sample by averaging $\sim 260$ NPs from the TEM images using ImageJ software.

The influence of US-treatment on the sizes and distribution of NPs was studied by using Atomic Force Microscopy (AFM, Solver Nano NT-MDT ISN, Laboratory of "Heliotechnics", National Polytechnic University of Armenia, Yerevan).

\section{Assessment of antibacterial activity}

The study of antibacterial activity of GS and ultrasound-treated Ag NPs was performed on S. typhimurium MDC1759 and S. aureus MDC5233 strains (Microbial Depository Center, National Academy of Science, Yerevan, Armenia). The mentioned strains were grown in Nutrient Broth (NB) media at $37^{\circ} \mathrm{C}$ and $\mathrm{pH}$ 7.5 (Gabrielyan et al. 2020). Anaerobic conditions were maintained as described (Gabrielyan et al. 2020). These strains were cultivated in the presence of GS and ultrasonicated Ag NPs (from 5 to $20 \mu \mathrm{L} \mathrm{mL}^{-1} \mathrm{v} / \mathrm{v}$ ratio). The growth of bacteria was measured by densitometer (DEN-1B McFarland, Biosan, Latvia) for $6 \mathrm{~h}$ of growth. The specific growth rate of bacteria was calculated using the following formula: growth rate $=\left(\operatorname{lnOD}_{t}-\operatorname{lnOD}_{0}\right) / t$, where $\mathrm{OD}_{0}$ (optical density) is the initial value of $\mathrm{OD}_{600}$; $\mathrm{OD}_{t}$ is the value of $\mathrm{OD}_{600}$ after $t$ hours (Gabrielyan et al. 2020).

\section{Statistical analysis}

Experiments were repeated three times. The results are represented as means $\pm \mathrm{SD}$. Standard errors, as well as the validity of the differences between different series of experiments, were evaluated by Student's validity criteria $(P)$, were calculated by using the appropriate functions of Microsoft Excel 2010.

\section{Results}

\section{UV-Vis spectroscopy analysis}

Change in color of the RJ solution mixed with $\mathrm{AgNO}_{3}$ solution from yellowish to brownish color visually observed, indicating Ag ions' reduction (Fig. 2a, b). After $24 \mathrm{~h}$ stirring final solution was centrifuged and washed with distilled water several times. Absorption measurements by UV-Vis spectrophotometer revealed the maximum absorption at $\sim 430 \mathrm{~nm}$, confirming the presence of Ag NPs (Fig. 3a).

\section{Fluorescence spectroscopy}

The fluorescence emission spectra of GS Ag nanostructures were obtained using from 370 to $420 \mathrm{~nm}$ excitation wavelengths, which are indicated on corresponding curves (Fig. 3b). Emission peak at $487 \mathrm{~nm}$ confirmed the presence of Ag nanostructures. By the increase of excitation wavelength, redshifted additional emission peaks were observed, which may be conditioned by the polydispersity of the NPs sample.

\section{Electron microscopy}

SEM and TEM were used to examine the morphology and microstructure of synthesized particles. Scanning electron micrograph revealed more or less spherical morphology of GS Ag NPs and showed that particles are coated by polymer (bright dots on SEM micrographs; Fig. 4a, b).

The analysis of data from TEM micrographs provided further insight into the morphology and particle size. It confirmed the formation of polydispersed and spherical

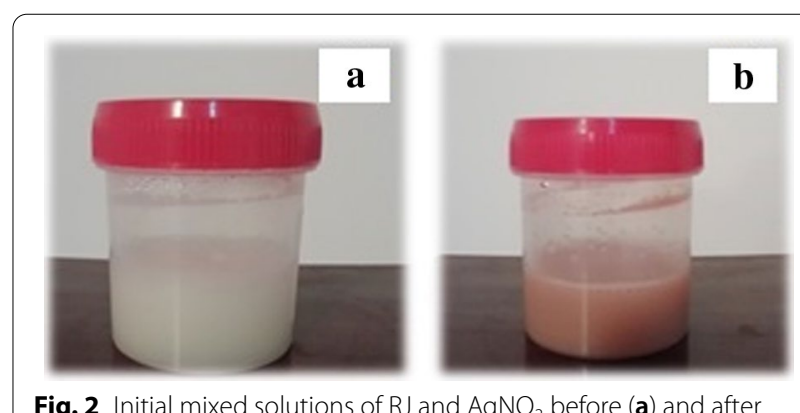

Fig. 2 Initial mixed solutions of $\mathrm{RJ}$ and $\mathrm{AgNO}_{3}$ before (a) and after incubation (b) 

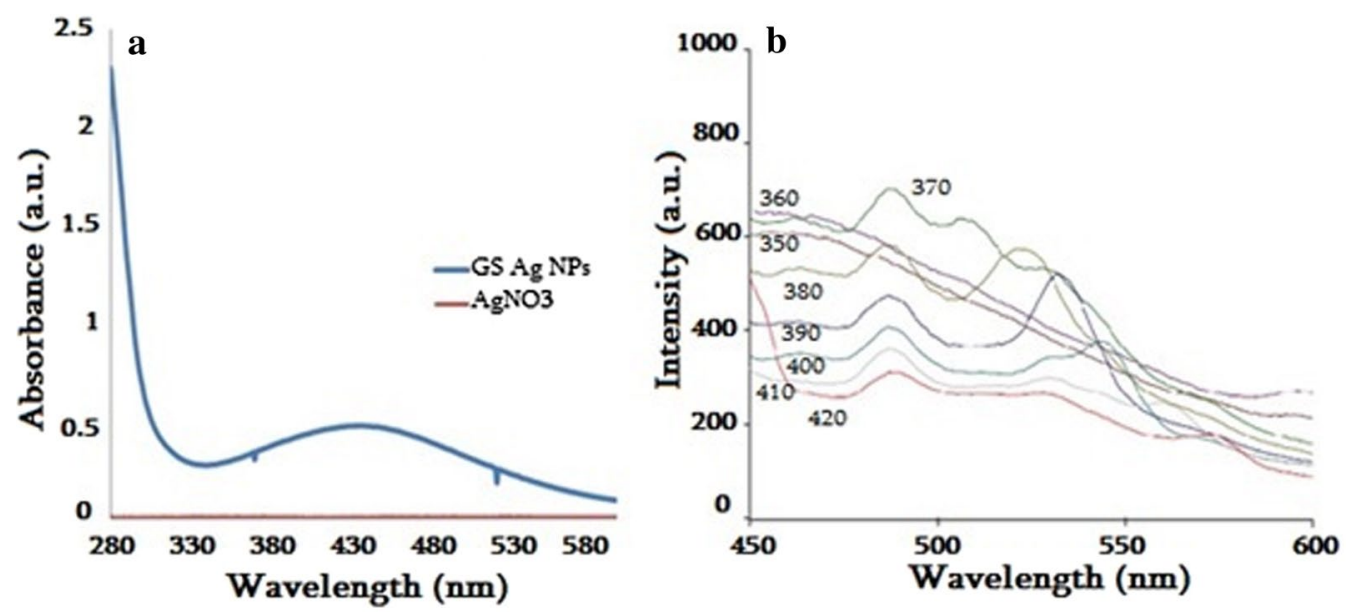

Fig. 3 UV-Vis absorption spectra (a) and fluorescence emission spectra (b) of GS Ag NPs
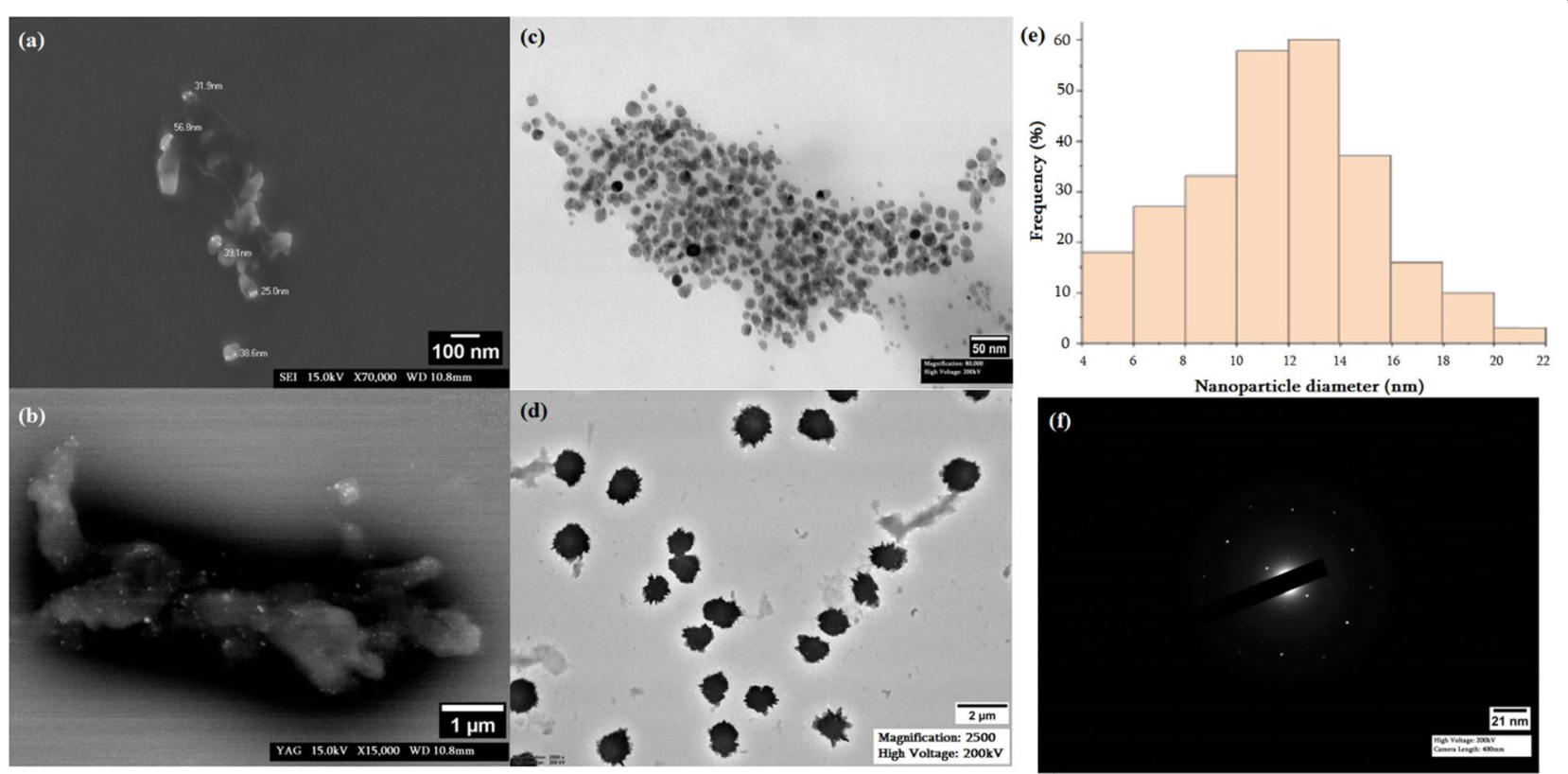

Fig. 4 SEM (a, b), TEM (c, d) micrographs and nanoparticle diameter distribution (e) and SAED pattern (f)

nanostructures of different sizes (Fig. 4c, d). Two different sample species could be identified. A small size species of GS Ag NPs with a particle size distribution are shown in the histogram in Fig. 4e. The average diameter of the spherical NPs was $11.7 \pm 3.58 \mathrm{~nm}$. And a second species containing a few large clusters up to $1 \mu \mathrm{m}$ was observed (see Fig. 4d), which can be a result of the aggregation of small nanostructures.

\section{Atomic force microscopy}

The effect of ultrasound treatment on GS Ag NPs was analyzed by AFM, and obtained data revealed that ultrasonication contributes to the homogenization of Ag NPs' colloidal solution and reduces the size of nanostructures (Fig. 5). For the purposes of comparative analysis, AFM images of Ag NPs without and with ultrasound-treatment as well as the size of the 

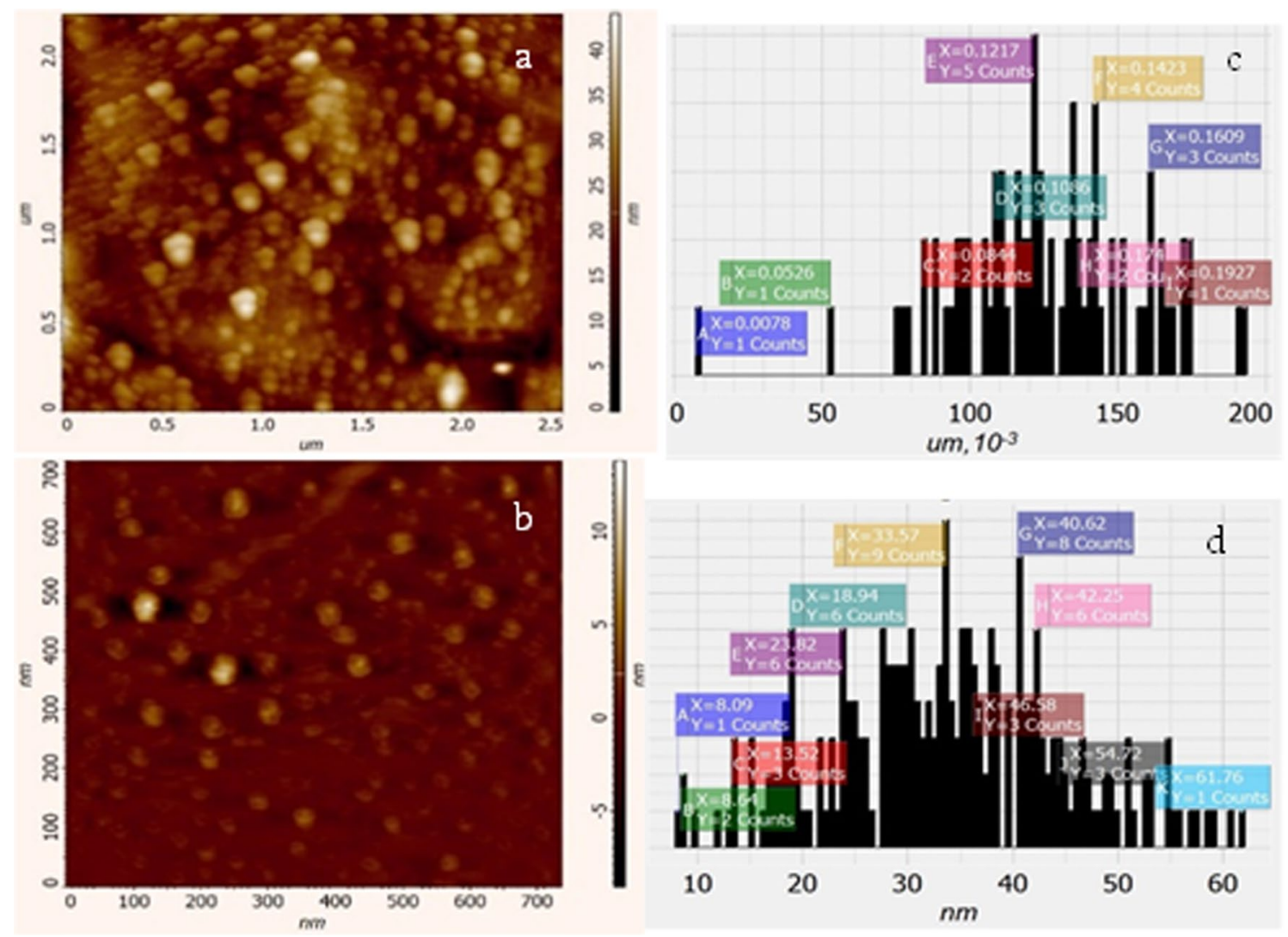

Fig. 5 AFM images of non-treated (ultrasound-treated $\mathbf{a}(\mathbf{b})$ ) and average size distribution $\mathbf{c}(\mathbf{d})$ of Ag NPs

corresponding nanoparticles distributions are shown (see Fig. 5), representing 2D topography of the NPs without and with US treatment, respectively. The data shows the distributions of particle sizes both without and with US treatment cases. Statistical analysis shows that before and after US-treatment NPs have sizes of $7.8-192.7 \mathrm{~nm}$ and $8.6-61.8 \mathrm{~nm}$, respectively. Furthermore, over $90 \%$ of the NPs have sizes in the interval of $52-174.0 \mathrm{~nm}$ and $8.9-54.7 \mathrm{~nm}$, respectively. The average values of some topographical parameters are presented in Table 1 . As a result, it can be said that US-treatment leads to a more homogeneous distribution and a significant reduction in the size of NPs.

\section{Antibacterial activity}

The growth properties of S. typhimurium MDC1759 and S. aureus MDC5233 strains, cultivated under anaerobic conditions, in the presence of GS and ultra-sonicated Ag NPs (from 5 to $20 \mu \mathrm{L}$ per $\mathrm{mL}$ ) have been investigated. The control was bacteria grown without NPs addition. The bactericidal effect for both bacteria was observed at all concentrations of NPs (Figs. 6, 7). Moreover, RJ-mediated Ag NPs display more expressed antimicrobial effect at low concentration (Fig. 6). Similar result was obtained with S. typhimurium MDC1759 in the presence of commercial Ag NPs (Gabrielyan et al. 2020).

The growth data analysis showed that Gram-negative $S$. typhimurium had higher sensitivity than Gram-positive $S$. aureus, which may be conditioned by the membrane

Table 1 Average topographical (error values: $\pm 5 \%$ ) parameters of GS Ag NPs with and without US-treatment

\begin{tabular}{lccccc}
\hline Ag NPs & Size, Nm & Length, Nm & Height, $\mathrm{nm}$ & Area nm*nm & Volume $\mathrm{nm}^{*} \mathrm{~nm}{ }^{*} \mathrm{~nm}$ \\
\hline Without US-treatment & 126.010 & 191.051 & 24.12 & $16,801.001$ & $129 \times 10^{6}$ \\
With US-treatment & 34.003 & 53.068 & 13.03 & 1279.048 & 3385.355 \\
\hline
\end{tabular}




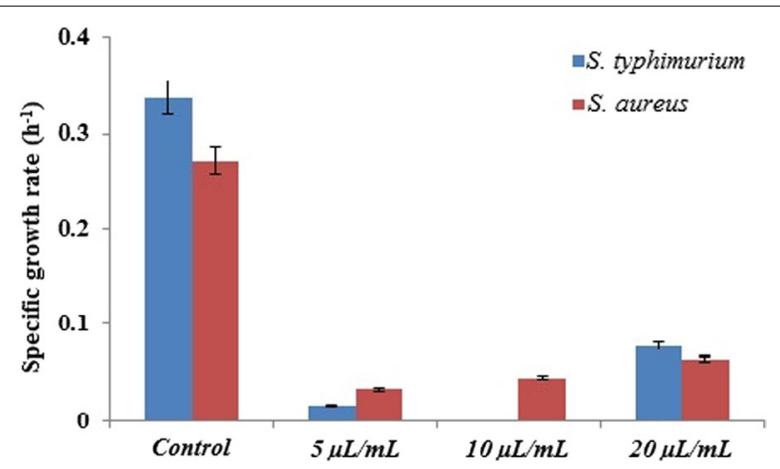

Fig. 6 Specific growth rate of S. typhimurium MDC1759 and S. aureus MDC5233, cultivated without (control) and with addition of different concentrations of GS Ag NPs $(p<0.05)$

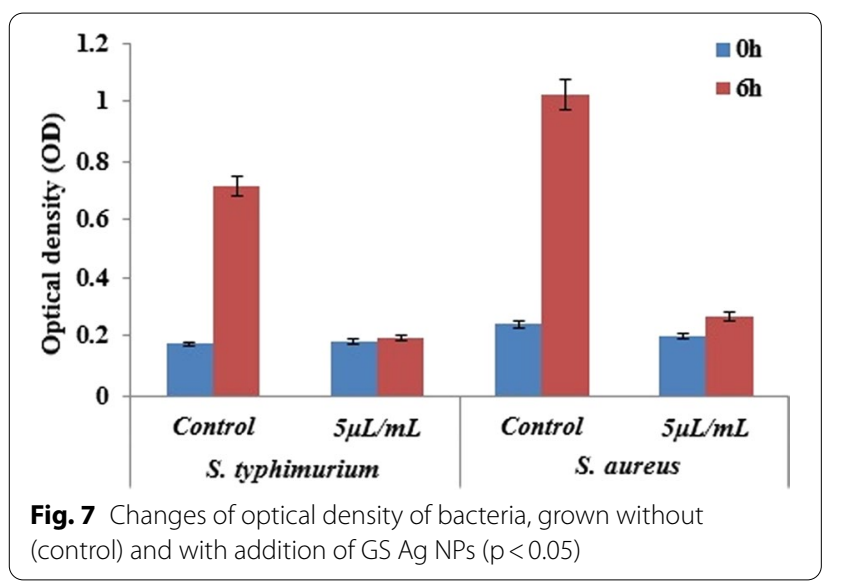

structure-a narrower cellular wall of these bacteria (Aghajanyan et al. 2020; Meikle et al. 2020). The antibacterial activity of RJ-mediated GS Ag NPs can be a result of interaction of NPs with bacterial membranes and their penetration into the bacterial cell (Trchounian et al. 2018; Gabrielyan et al. 2020).

\section{Discussion}

It is well known that $\mathrm{Ag}$ ions and Ag-based compounds, including Ag NPs, exhibit a toxic effect on both Gramnegative and Gram-positive microorganisms (Losasso et al. 2014). The exact mechanism of antibacterial activity of Ag NPs has not been entirely clarified, but various hypotheses have been proposed. The action of Ag NPs can be conditioned by free Ag ions, present or released from the nanomaterials, which can bind to the cell membrane, destabilize membrane potential and lead to disruption of the bacterial envelope (Bapat et al. 2018; Yin et al. 2020). Free silver ions also can induce the generation of reactive oxygen species as well as inhibit the synthesis of proteins by denaturing ribosomes (Durán et al. 2016). Different capping agents, including biologically active compounds as well as the presence of organic and inorganic components in media, can change the dissolution behavior of NPs, thus influencing the release of silver ions and antibacterial activity strength of Ag NPs (Yin et al. 2020). According to Konovalov et al. (2013), biologically active compounds (BACs) as a capping agent can have a great impact on efficient concentration. BACs, especially in the case of GS of Ag NPs, can lead to the formation of nanoassociates in highly diluted samples, which will increase the efficiency. This may also be conditioned by the higher diffusion rate of low concentrated solutions compared to highly concentrated solutions, which can form aggregates (Konovalov et al. 2013). On the other hand, antimicrobial activities of many peptides present in RJ were demonstrated (Romanelli et al. 2011; Li et al. 2012). The action mechanism of these antimicrobial peptides is due to the change of cell membrane permeability; particularly they cause decrease of lipid layer surface and lead to the membrane disruption or create pores thus destabilizing membrane (Fratini et al. 2016; Li et al. 2012). Cell wall structure also plays a crucial role in the antibacterial activity of Ag NPs. Gram-negative bacteria are more susceptible to Ag NPs due to narrower cellular walls compared to Gram-positive strains (Aghajanyan et al. 2020; Meikle et al. 2020).

TEM revealed the presence of small as well as clustered NPs. US-treatment leads to a decrease in the size and an increase in homogeneity of the distribution of GS NPs and contributes to the higher antibacterial activity against Gram-negative S. typhimurium and Gram-positive $S$. aureus, in that Gram-negative was more susceptible. The results suggest that green synthesis plays an important role in optimizing properties and biological activity as well as the inhibitory efficiency of synthesized Ag NPs, while to better understand the antibacterial activity mechanism of GS Ag NPs, further research is needed.

\section{Abbreviations}

GS: Green synthesis; RJ: Royal jelly; Ag NPs: Silver nanoparticles; SEM: Scanning electron microscopy; TEM: Transmission electron microscopy; AFM: Atomic force microscopy; US: Ultrasonication; SAED: Selected area electron diffraction; OD: Optical density; MDC: Microbial depository center.

\section{Acknowledgements}

We thank to Dr. Stepan Tatikian (CANDLE Synchrotron Research Institute, Yerevan, Armenia) and PhD student Henrik Parsamian (Yerevan State University, Armenia) for help in some experiments and "Royal Jelly" LTD for supplying Royal Jelly for experiments. This work was performed by the support of CANDLE Synchrotron Research Institute, Armenia; National Polytechnic University of Armenia; Leibniz Institute of Photonic Technologies, Germany; European XFEL GmbH, Schenefeld, Germany, in particular the use of the XBI biological sample preparation laboratory, enabled by the XBI User Consortium.

\section{Authors' contributions}

SG performed the main part of experimental work, analysed the data and prepared the manuscript; RS provided TEM study of NPs, edited and revised 
the manuscript; MY provided AFM of NPS, edited and revised the manuscript; LG supported the antibacterial activity assays and data analysis, edited and revised the manuscript; KL participated in the discussions and made critical comments; AT and KT supervised the study and edited the manuscript. All authors read and approved the final manuscript.

\section{Funding}

This work was partially supported by SC MES RA, within the frame of the research Project No. 18T-1C247, ANSEF research Grant (condmatth-5214), Faculty Research Funding Program EIF and PMI Science.

\section{Availability of data and materials}

All data generated or analysed during this study are included in this article.

\section{Declarations}

\section{Ethics approval and consent to participate}

The article does not contain any studies with humans and animals.

\section{Consent for publication}

Not applicable.

\section{Competing interests}

The authors declare that they have no competing interests.

\section{Author details}

${ }^{1}$ Department of Biochemistry, Microbiology and Biotechnology, Yerevan State University, Alex Manoogian 1, 0025 Yerevan, Armenia. ${ }^{2}$ European X-ray Free Electron Laser GmbH, Holzkoppel 4, 22869 Schenefeld, Germany. ${ }^{3}$ Institute of Chemical Physics, NAS RA, Paruir Sevak 5/2, 0014 Yerevan, Armenia. ${ }^{4}$ Military Aviation University Named After Marshal A. Khamperyants, Arshakunyats 89, 0007 Yerevan, Armenia.

Received: 23 January 2021 Accepted: 24 March 2021

Published online: 01 April 2021

\section{References}

Aghajanyan A, Gabrielyan L, Schubert R, Trchounian A (2020) Silver ion bioreduction in nanoparticles using Artemisia annua L. extract: characterization and application as antibacterial agents. AMB Express 10:66. https:// doi.org/10.1186/s13568-020-01002-w

Bapat RA, Chaubal TV, Joshi CP, Bapat PR, Choudhury H, Pandey M, Gorain B, Kesharwani P (2018) An overview of application of silver nanoparticles for biomaterials in dentistry. Mater Sci Eng C 91:881-898. https://doi.org/10. 1016/j.msec.2018.05.069

Durán N, Durán M, de Jesus MB, Seabra AB, Fávaro WJ, Nakazato G (2016) Silver nanoparticles: a new view on mechanistic aspects on antimicrobial activity. Nanomed 12(3):789-799. https://doi.org/10.1016/j.nano.2015.11.016

Forough M, Farhadi K (2010) Biological and green synthesis of silver nanoparticles. Turkish J Eng Env Sci 34(4):281-287

Fratini F, Cilia G, Mancini S, Felicioli A (2016) Royal jelly: an ancient remedy with remarkable antibacterial properties. Microbiol Res 192:130-141. https:// doi.org/10.1016/j.micres.2016.06.007

Gabrielyan L, Badalyan H, Gevorgyan V, Trchounian A (2020) Comparable antibacterial effects and action mechanisms of silver and iron oxide nanoparticles on Escherichia coli and Salmonella typhimurium. Sci Rep 10:13145. https://doi.org/10.1038/s41598-020-70211-x

Gabrielyan L, Trchounian A (2019) Antibacterial activities of transient metals nanoparticles and membranous mechanisms of action. World J Microb Biot 35:162. https://doi.org/10.1007/s11274-019-2742-6

Ge L, Li Q, Wang M, Ouyang J, Li X, Xing M (2014) Nanosilver particles in medical applications: synthesis, performance, and toxicity. Int J Nanomed 9:2399-2407. https://doi.org/10.2147/IJN.S55015

Guo H, Saiga A, Sato M, Miyazawa I, Shibata M, Taka Hata Y, Morimatsu F (2007) Royal jelly supplementation improves lipoprotein metabolism in humans. J Nutr Sci Vitaminol 53(4):345-348. https://doi.org/10.3177/jnsv.53.345
Han H, Round E, Schubert R, Gü Y, Makroczyova J, Meza D, Heuser P, Aepfelbacher M, Bara I, Betzel C, Fromme P, Kursula I, Nissen P, Tereschenko E, Schulz J, Uetrecht C, Ulicny J, Wilmanns M, Hajdu J, Lamzinc VS, Lorenzen K (2021) The XBI BioLab for life science experiments at the European XFEL. J Appl Cryst 54(1):7-21. https://doi.org/10.1107/S16005767200139 89

Ismail M, Khan MI, Akhtar K, Khan MA, Asiri AM, Khan BS (2018) Biosynthesis of silver nanoparticles: a colorimetric optical sensor for detection of hexavalent chromium and ammonia in aqueous solution. Phys E Low Dimens Syst Nanostruct 103:367-376. https://doi.org/10.1016/j.physe.2018.06.015

Joerger R, Klaus T, Granqvist CG (2000) Biologically produced silver-carbon composite materials for optically functional thin-film coatings. Adv Mater 12:407-409

Kolayli S, Sahin H, Can Z, Yildiz O, Malkoc M, Asadov A (2015) A member of complementary medicinal food: anatolian royal jellies, their chemical compositions, and antioxidant properties. Evid Based Complement Alternat Med 21(4). https://doi.org/10.1177/2156587215618832.

Konovalov Al (2013) The formation of nanosized molecular ensembles in highly dilute aqueous solutions. Her Russ Acad Sci 83:513-519. https:// doi.org/10.1134/S1019331613060099

Kozma G, Rónavári A, Kónya Z, Kukovecz Á (2016) Environmentally benign synthesis methods of zero-valent iron nanoparticles. ACS Sustain Chem Eng 4(1):291-297. https://doi.org/10.1021/acssuschemeng.5b01185

Lara HH, Garza-Treviño EN, Ixtepan-Turrent L, Singh DK (2011) Silver nanoparticles are broad-spectrum bactericidal and virucidal compounds. J Nanobiotechnol 9:30. https://doi.org/10.1186/1477-3155-9-30

Li Y, Xiang Q, Zhang Q, Huang Y, Su Z (2012) Overview on the recent study of antimicrobial peptides: origins, functions, relative mechanisms and application. Peptides 37(2):207-215. https://doi.org/10.1016/j.peptides. 2012.07.001

Losasso C, Belluco S, Cibin V, Zavagnin P, Mičetić I, Gallocchio F, Zanella M, Bregoli L, Biancotto G, Ricci A (2014) Antibacterial activity of silver nanoparticles: sensitivity of different Salmonella serovars. Front Microbiol 5:227. https://doi.org/10.3389/fmicb.2014.00227

Meikle TG, Dyett BP, Strachan JB, White J, Drummond CJ, Conn CE (2020) Preparation, characterization, and antimicrobial activity of cubosome encapsulated metal nanocrystals. ACS Appl Mater Interfaces 12(6):69446954. https://doi.org/10.1021/acsami.9b21783

Mendoza-Reséndez R, Gomez-Treviño A, Barriga-Castro ED, Núñez NO, Luna C (2014) Synthesis of antibacterial silver-based nanodisks and dendritic structures mediated by royal jelly. RSC Adv 4(4):1650-1658. https://doi. org/10.1039/C3RA45680C

Moghaddam AB, Namvar F, Moniri M, Tahir PM, Azizi S, Mohamad R (2015) Nanoparticles biosynthesized by fungi and yeast: a review of their preparation, properties, and medical applications. Molecules 20(9):1654016565. https://doi.org/10.3390/molecules 200916540

Nagai T, Inoue R (2004) Preparation and the functional properties of water extract and alkaline extract of royal jelly. Food Chem 84:181-186. https:// doi.org/10.1016/S0308-8146(03)00198-5

Narayanan KB, Sakthivel N (2011) Green synthesis of biogenic metal nanoparticles by terrestrial and aquatic phototrophic and heterotrophic eukaryotes and biocompatible agents. Adv Colloid Interface Sci 169(2):59-79. https://doi.org/10.1016/.cis.2011.08.004

Rónavári A, Kovács D, Igaz N, Vágvölgyi C, Boros IM, Kónya Z, Pfeiffer I, Kiricsi M (2017) Biological activity of green-synthesized silver nanoparticles depends on the applied natural extracts: comprehensive study. Int J Nanomedicine 12:871-883. https://doi.org/10.2147/IJN.S122842

Romanelli A, Moggio L, Montella RC, Campiglia P, lannaccone M, Capuano F, Pedone C, Capparelli R (2011) Peptides from royal jelly: studies on the antimicrobial activity of jelleins, jelleins analogs and synergy with temporins. J Pept Sci 17(5):348-352. https://doi.org/10.1002/psc.1316

Salem SS, Fouda MMG, Fouda A, Awad MA, Al-Olayan EM, Allam AA, Shaheen TI (2020) Antibacterial, cytotoxicity and larvicidal activity of green synthesized selenium nanoparticles using penicillium corylophilum. J Clust Sci 32:351-361. https://doi.org/10.1007/s10876-020-01794-8

Sugiyama T, Takahashi K, Mori H (2012) Royal jelly acid, 10-hydroxy-trans2-decenoic acid, as a modulator of the innate immune responses. Endocr Metab Immune Disord Drug Targets 12(4):368-376. https://doi.org/10. 2174/187153012803832530

Swellam T, Miyanaga N, Onozawa M, Hattori K, Kawai K, Shimazui T, Akaza H (2003) Antineoplastic activity of honey in an experimental bladder cancer 
implantation model: in vivo and in vitro studies. Int J Urol 10:213-219. https://doi.org/10.1046/j.0919-8172.2003.00602.x

Thunugunta T, Reddy AC, Reddy DCL (2015) Green synthesis of nanoparticles: current prospectus. Nanotechnol Rev 4(4):303-323. https://doi.org/10. 1515/ntrev-2015-0023

Trchounian A, Gabrielyan L, Mnatsakanyan N (2018) Nanoparticles of various transition metals and their applications as antimicrobial agents. In: Saylor Y, Irby V (eds) Metal nanoparticles: properties, synthesis and applications. Nova Science Publication, New York

Venu R, Ramulu TS, Anandakumar S, Rani VS, Kim CG (2011) Bio-directed synthesis of platinum nanoparticles using aqueous honeysolutions and their catalytic applications. Colloids Surf A 384:733-738. https://doi.org/ 10.1016/j.colsurfa.2011.05.045
Vishwarsrao C, Momin B, Ananthanarayan L (2019) Green synthesis of silver nanoparticles using sapota fruit waste and evaluation of their antimicrobial activity. Waste Biomass Valor 10:2353-2363. https://doi.org/10.1007/ s12649-018-0230-0

Yin IX, Zhang J, Zhao IS, Mei ML, Li Q, Chu CH (2020) The antibacterial mechanism of silver nanoparticles and its application in dentistry. Int J Nanomed 15:2555-2562. https://doi.org/10.2147/IJN.S246764

\section{Publisher's Note}

Springer Nature remains neutral with regard to jurisdictional claims in published maps and institutional affiliations.

\section{Submit your manuscript to a SpringerOpen ${ }^{\odot}$ journal and benefit from:}

- Convenient online submission

- Rigorous peer review

- Open access: articles freely available online

- High visibility within the field

- Retaining the copyright to your article

Submit your next manuscript at $>$ springeropen.com 